\title{
Multilevel spatio-temporal dual changepoint models for relating alcohol outlet destruction and changes in neigh- bourhood rates of assaultive violence
}

\author{
Qingzhao $\mathrm{Yu}^{1}$, Richard Scribner ${ }^{1}$, Brad Carlin², Katherine Theall ${ }^{1}$, Neal Simonsen ${ }^{1}$, \\ Bonnie Ghosh-Dastidar ${ }^{3}$, Deborah Cohen ${ }^{3}$, Karen Mason ${ }^{1}$ \\ ${ }^{1}$ School of Public Health, Lomisiana State University Health Sciences Center, New Orleans, LA, USA; \\ ${ }^{2}$ School of Public Health, University of Minnesota, Minneapolis, MN, USA; ${ }^{3}$ RAND Corporation, Santa \\ Monica, CA, USA
}

\begin{abstract}
Many previous studies have suggested a link between alcohol outlets and assaultive violence. In this paper, we evaluate the impact of the "1992 Civil Unrest" in Los Angeles (which followed the "Rodney King incident"), in which many alcohol outlets were damaged leading to a decrease in alcohol outlet density, on crime. We leverage the natural experiment created by the closure of alcohol outlets in certain areas and not others to explore the effects of alcohol availability on assault crimes at the census tract level. We develop a hierarchical model that controls for important covariates (such as race and socio-economic status) and accounts for unexplained spatial and temporal variability. While our model is somewhat complex, its hierarchical Bayesian analysis is accessible via the WinBUGS software. Our results show that, keeping other effects fixed, the reduction in alcohol availability within a census tract was associated with a drop in the assaultive violence rate at the census tract level. Comparing several dual candidate changepoint models using the Deviance Information Criterion, the drop in assaultive violence rate is best seen as having occurred one year after the reduction in alcohol availability, with the effect lasting roughly five years. We also create maps of the fitted assault rates in Los Angeles, as well as spatial residual maps that suggest various spatially-varying covariates are still missing from our model.
\end{abstract}

Keywords: assault trend, conditionally autoregressive (CAR) model, Gibbs sampler, natural experiment, neighbourhood.

\section{Introduction}

The growing body of literature on the contextual role of alcohol outlets on a variety of health outcomes including assaultive violence over the past two decades has been paralleled by a number of methodological innovations. Since the effect of alcohol outlets is believed to be contextual, these analyses involve areal data that require more sophisticated techniques to account for their spatial and tem-

Corresponding author:

Qingzhao Yu

LSUHSC School of Public Health

Suite 1400, 1615 Poydras Street, New Orleans, LA 70112, USA

Tel. +1 504568 6086; Fax +1 5045686075

E-mail: qyu@1suhsc.edu poral structure. This study takes the next step in this methodological progression by constructing a Bayesian framework to address these issues.

Initial studies on the role of alcohol outlets in the neighbourhood environment and assaultive violence were ecological in design and conducted at the city level (Watts and Rabow, 1983; Scribner et al., 1995). As geographical information systems (GIS) software became widely available, more local units of analysis (e.g., census tracts) were used when modeling the theoretical relation between alcohol outlets in a neighbourhood and assaultive violence (Speer et al., 1998; Scribner et al., 1999). Accounting for spatial autocorrelation, i.e. the possible spillover relation between outlets and assaultive violence into contiguous neighborhoods, was a subsequent 
advancement (Gruenewald, 2000; Gorman et al., 2001; Zhu et al., 2004). Recently, longitudinal models have been incorporated into the analysis of the data, adding an additional level of complexity (Gruenewald and Remer, 2006). In this study we apply a Bayesian spatio-temporal model to temporal changes in assaultive violence rates among census tracts affected by the "1992 Civil Unrest" in Los Angeles which resulted in the immediate loss of over 250 alcohol outlets, and the permanent loss of roughly 150 . We will also model the spatial dependence of rates of assaultive violence on the conditions in contiguous tracts by accounting for spatial error in the residuals.

Bayesian methods have become popular tools in subject areas including population epidemiology, public policy, environmental science, and criminology since the methods can properly account for the spatial as well as the temporal dependence of the estimates. Besag (1974) described a probability model, the conditionally autoregressive (CAR) model, that accounts for spatial structure in neighbouring geographic units. This was extended by Besag et al. (1991) to separate spatial variability from random heterogeneity in regional disease maps. Britt et al. (2005) and Zhu et al. (2006) examined the relationship between alcohol outlet density and violence in different areas using hierarchical Bayesian spatial models. In this paper, we add a dual temporal changepoint dimension to the modeling in order to analyze trends in assaultive violence in Los Angeles at the census tract-level. We investigate whether alcohol outlet density is related to assaultive violence, taking advantage of the "1992 Civil Unrest" as a natural experiment. These analyses add to the growing literature within public health and criminology about the geospatial relationship between aspects of the physical and social environment and the incidence of violent crime or the spread of disease.

The remainder of our paper is organized as follows. Firstly, we describe the "1992 Civil Unrest" that defines our natural experiment, as well as the datasets used to characterize alcohol outlets, assaultive violence and socio-demographic characteristics of the census tracts in the city of Los Angeles affected by the civil unrest in the years before and after 1992. Secondly, we present the spatio-temporal model that was used to analyze the assault trend in Los Angeles, accounting for socioeconomic factors as well as spatial correlations among the neighbours at a census tract level. The models are compared using the Deviance Information Criterion (DIC; Spiegelhalter et al., 2002). Thirdly, we report the results of the DIC-best model, and interpret them in the context of our natural experiment. Maps are constructed to investigate the distribution of fitted assault rates in Los Angeles, and to show how excess spatial heterogeneity is allocated by the Bayesian hierarchical model. Finally, we discuss our findings and points out areas for future work.

\section{Materials and methods}

A natural experiment in reducing alcohol availability

Our study is designed to capitalize on a natural experiment. The experiment was initiated by the civil unrest in Los Angeles that followed the verdicts acquitting the police officers accused of beating Rodney King. The riots resulted in 53 deaths, 2,325 reported injuries, more than 600 buildings completely destroyed by fire, and approximately 735 million US dollars in total damages (Evans, 1993). Many of the buildings destroyed were serving as alcohol purchase outlets. As a result, a total of 279 liquor licenses were surrendered across 144 census tracts in Los Angeles County due to interruption of their services. In the wake of the civil unrest, an effort to halt the rebuilding of off-sale alcohol outlets was successful in restricting the relicensing of outlets with a history of problems (e.g., assaults, homicides, drug sales) around their premises. The effort was responsible, in part, for over 100 outlets permanently closing in the civil unrest area. These events provide a natural experimental setting to test various hypotheses regarding the 
effects of closure of off-sale liquor outlets in 144 tracts, compared to 336 tracts also exposed to the civil unrest where outlets were not affected (Cohen et al., 2006).

\section{Study area}

The "1992 Civil Unrest" occurred over a large area of South Central Los Angeles. In the present study, we include only those census tracts that suffered material damage in the civil unrest, thereby controlling for a possible global effect of the unrest on outcomes. That is, any measured effect associated with specific temporal changes in a tract's neighbourhood environment should be independent of any global effect of the civil unrest, which would affect all tracts regardless of the presence or absence of changes in their neighbourhood alcohol environment. To define the study area in this manner, we used the definition established by the Rose Institute of California State and Local Government at Claremont McKenna College (http://ccdl.libraries.claremont.edu/col/ric/) to study the economic impact of the civil unrest (Hubler, 2002). A total of 480 census tracts comprise the unrest area. These tracts contained $2,641,320$ people in 1990 , of whom $48 \%$ were Hispanic and $27 \%$ were African American. A total of 2,240 unique addresses were damaged in the 480 tracts, while 144 tracts had one or more offsale liquor outlets whose license was surrendered. The majority of the damaged addresses were commercial businesses. Immediately following the civil unrest, there emerged a grass roots effort among the affected communities to halt the rebuilding of alcohol outlets based on the finding that an overconcentration of off-sale outlets existed in the low socio-economic status areas prior to the civil unrest (Berestein, 1994; Kang, 1994; Grills et al., 1996).

The study time frame is 1990 to 1999 . Annual counts of liquor outlet licenses came from the California Department of Alcohol Beverage Control $(\mathrm{ABC})$. A list of outlets that surrendered their licenses and a list of stores with riot damage as a result of the "1992 Civil Unrest" of Los Angeles were also obtained from the ABC. Alcohol outlets were classified based on their license to sell alcohol for onpremise (bars and restaurants) or off-premise (liquor stores, grocery stores, and convenience stores) consumption using license codes provided by the ABC. Information on damaged buildings (Ong, 1993) came, directly or indirectly, from four different sources: the Los Angeles City Department of Building and Safety, the Korean Central Daily, the California Insurance Commission, and the Compton Department of Building and Safety. The annual estimates of population distributions by age, race and sex were obtained from the Los Angeles County Department of Health Services, with actual counts available for 1990 and 1995 and counts for the other years estimated from birth and death records. The remaining socio-demographic data were obtained from the 1990 U.S. Census data of Los Angeles County.

All unique address listings were geo-coded and mapped to the 1990 Census tract areas, and individual data sources were matched by census tract. Ninety-eight percent of addresses could be matched using Arcview 3.2 GIS software (ESRI Inc, Redlands, CA, USA) with Los Angeles County Topographically Integrated Geographic Encoding and Referencing (TIGER) street files from the 2000 census. Addresses that the computer was unable to match were hand placed with the help of an Internet mapping site (Mapquest) and a Thomas Guide map book.

\section{Assaultive violence}

Our measure of assaultive violence was obtained from the Los Angeles Police Department. Uniform Crime Report (UCR) offenses involving assaultive violence (i.e., murder, rape, robbery, and assault) were obtained for the years 1990 through 1999. A summary measure of the count of all violent offenses was generated for each census tract and for all study years by geo-coding the data that contained the street address of the offense location. 


\section{Alcohol outlet density}

Measures of alcohol exposure included surrender of off-sale liquor outlet licenses following the "1992 Civil Unrest", the percentage of off-sale liquor outlet licenses surrendered, and annual off-sale outlet counts or outlet density from 1990 to 1999. From data on outlet surrenders two tract-level measures were incorporated in the analysis to assess the effect of decreased alcohol availability due to outlet destruction during the "1992 Civil Unrest". The measures include:

(i) tracts with at least one license surrendered - a dichotomous indicator of census tracts that did and did not have outlet licenses surrendered due to destruction; and

(ii) percent surrendered - a continuous measure indicating the percentage of all off-sale outlet licenses that were surrendered in a tract, denoted as "\% offsale surrender" in our analysis.

Annual alcohol outlet density was measured as the ratio of the number of off-sale liquor outlets to the number of miles of roadway in each tract based on 1990 geographic census data, denoted as "Alcohol density". The variable was used to quantify exposure to alcohol outlets. The number per capita and per square mile area were also calculated and yielded results consistent with those for density per road mile.

\section{Additional covariates}

(i) Heterogeneity in race. These covariates represent the proportion of residents in the census tract that are non-white. The covariates are calculated for each year of observation by dividing the population of blacks, Hispanics and Asians, respectively, by the total population in the census tract. We denote these variables as "\% Black", “\% Hispanic” and "\% Asian”.

(ii) Percentage of young males in the neighbourhood. This covariate is calculated by dividing the number of males between ages 15 and 30 by the total population in each census tract. This covariate is denoted by "\% Male 15 to 30 ".

(iii) “\% Households in poverty". This covariate captures the poverty level in each census tract. It is calculated by dividing the number of households in poverty by the total number of households in that census tract. Poverty is defined by the U.S. Census as a percentage of the population below a yearly income cutoff based on family size and the presence of family members under eighteen years of age. The income cutoffs increase as family size increases; for example, in 1990 the income level was 6,652 US dollars for one individual and 22,582 US dollars for all members of a family with 8 people of which 7 are children under 18 years of age.

The above covariates are annual estimates available for the years 1990-1999 and are included to control for changes in tract composition over time, an endogeneous change that could explain temporal changes in assaultive violence rates. For example, it is possible that changes in assaultive violence are the result of the movement of populations at higher or lower risk for violence into or out of particular study tracts over the course of the observation period.

(iv) Damage level. Physical damage is measured as a binary indicator of any damage of property in the tract due to the "1992 Civil Unrest". We also derived a measure of damage density (i.e. damage per square mile), "Damage per Square Mile", calculated as the ratio of the number of unique addresses damaged in the "1992 Civil Unrest" to the amount of land in the tract used for commercial purposes. The denominator corresponds to land used for commercial purposes because most of the damaged property was commercial. To compute the denominator, we estimated the proportion of commercial space in the tract using a land use file and multiplied it by the area of the tract in 1990 in square miles. In addition to these factors, we use "year", which ranges from 1990 to 1999, to measure the temporal trend in assaults. 
Spatio-temporal dual changepoint modeling

A spatio-temporal dual changepoint model was built to identify the relationship between crimes and alcohol availability. In the model, we explore the changing relationship over time while controlling for measured and unmeasured factors that might have an influence on the assaultive crime rates.

\section{Changepoint model}

To see a rough picture of the relationship between alcohol availability and assaultive crime, Figure 1 plots the average assault rate over the years 1990 to 1999. To investigate the impact of alcohol license surrender, we separately draw the crime rates in the tracts with and without license surrender following the "1992 Civil Unrest". We see that the tracts that had alcohol license surrender (i.e., a decrease in alcohol outlet density) after the civil unrest have a different temporal trend than the tracts that did not. Moreover, we can see that the assault rate is associated with this measure of alcohol availability.

Figure 1 shows that on average, the tracts that had alcohol license surrender after the "1992 Civil Unrest" have higher assault rates over the ten-year period. The differences in assault rates in the two groups appear to shrink after the civil unrest, and then stabilize a few years later. To model this relationship, suppose we allow different slopes in assault trends before and after the civil unrest in the census tracts that have alcohol license surrender, and also allow this slope to return to its pre-civil unrest level in the later years. Such changepoint models have attracted significant attention in the hierarchical modeling literature (Carlin et al., 1992; Green, 1995). The lines superimposed on Figure 1 show one possible dual changepoint model fit; our hierarchical model below will work on the log scale and account for observed and unobserved spatial covariates.

The "no surrender group" has only a single slope, set equal to the pre-civil unrest slope in the surrender group. If alcohol availability is positively associ- ated with the assault rate, compared with the census tracts that have no alcohol license surrender, there should be a steeper drop in assault rates for those tracts that had alcohol license surrender following the civil unrest. The effect might last a few years before the difference in crime rates between the two groups stabilizes. In the meantime, we should also consider other factors that might be associated with the assault rate.

To construct the dual changepoint models, we use an indicator variable ("indyear") that increases to some plateau during the years after the civil unrest. The key aspect of our model is actually the interaction between "indyear" and "\% offsale surrender". If the coefficient of this interaction term is significant, then compared with the tracts that had no alcohol license surrender following the "1992 Civil Unrest", the assault trend is altered significantly after the civil unrest for those census tracts that had alcohol license surrender, and in an amount proportional to the magnitude of this surrender. The change in trend persists through the years specified by "indyear," after which it is assumed to stabilize back to its pre-civil unrest level. To consider different locations for the two temporal changepoints, we fit several models using different definitions of

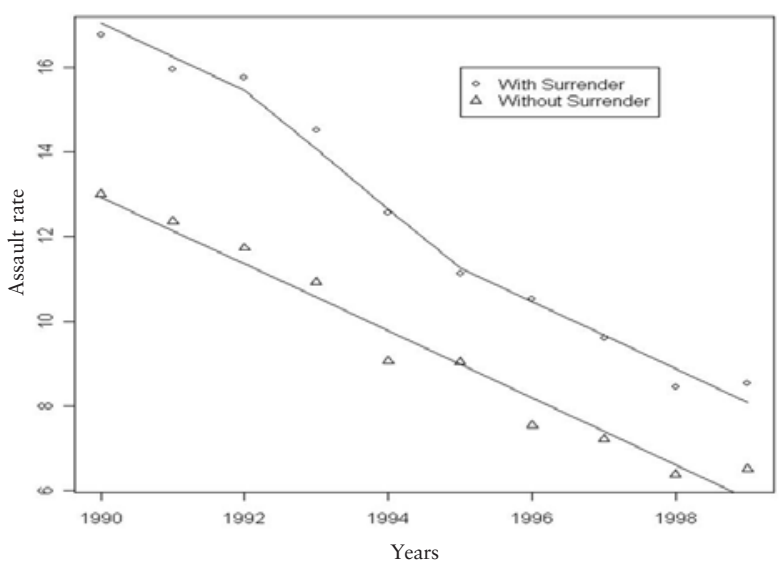

Fig. 1. Annual assault rates for tracts with and without alcohol outlet license surrender following the "1992 Civil Unrest" in Los Angeles. 
Table 1. Values of “indyear". Model 1 means a tract's level of alcohol license surrender has no significant effect on its assault rate; models 2 through 8 mean there is an effect and the effect lasts for one to seven years; and models 9 through 14 mean there is an effect, but it first takes effect one year after the surrender.

\begin{tabular}{|c|c|c|c|c|c|c|c|c|c|c|}
\hline \multirow[t]{2}{*}{ Model } & \multicolumn{10}{|c|}{ Indyear } \\
\hline & 90 & 91 & 92 & 93 & 94 & 95 & 96 & 97 & 98 & 99 \\
\hline 1 & 0 & 0 & 0 & 0 & 0 & 0 & 0 & 0 & 0 & 0 \\
\hline 2 & 0 & 0 & 0 & 1 & 1 & 1 & 1 & 1 & 1 & 1 \\
\hline 3 & 0 & 0 & 0 & 1 & 2 & 2 & 2 & 2 & 2 & 2 \\
\hline 4 & 0 & 0 & 0 & 1 & 2 & 3 & 3 & 3 & 3 & 3 \\
\hline$\cdots$ & \multicolumn{10}{|c|}{$\ldots$} \\
\hline 8 & 0 & 0 & 0 & 1 & 2 & 3 & 4 & 5 & 6 & 7 \\
\hline 9 & 0 & 0 & 0 & 0 & 1 & 1 & 1 & 1 & 1 & 1 \\
\hline 10 & 0 & 0 & 0 & 0 & 1 & 2 & 2 & 2 & 2 & 2 \\
\hline$\ldots$ & \multicolumn{10}{|c|}{$\cdots$} \\
\hline 14 & 0 & 0 & 0 & 0 & 1 & 2 & 3 & 4 & 5 & 6 \\
\hline
\end{tabular}

"indyear," with the "best" model being chosen according to the DIC criterion. Table 1 gives the values of "indyear" in the models. An illustration of the effect of the variable "indyear" is shown in Figure 1 in the case of Model 4. This model assumes that after the civil unrest, the assault rate experienced a steeper decrease in those tracts that had offsale license surrender, compared with those tracts that did not. The more dramatic decrease in assault rate lasts for three years, after which the rate returns to the pre-civil unrest rate. While this model offers good visual fit to the data, below we offer a more formal comparison of the models in Table 1.

\section{Model details and comparison}

We now describe the details of our spatio-temporal model. Let $\mathrm{y}_{\mathrm{i}}$ and $\mathrm{E}_{\mathrm{i}}$ be the observed and expected number of assaults in census tract-year combination i, where i ranges from 1 to 2900 since we have 290 census tracts and 10-years of data. The $\mathrm{E}_{\mathrm{i}}$ are the internally standardized expected counts of assault for each census tract-year pair, and are defined as the numbers at risk in $\mathrm{i}$ times the grand assault rate over all tracts and years. Our basic model assumes a
Poisson distribution of the counts of assaultive violence, with the following form:

$\mathrm{y}_{\mathrm{i}} \sim$ Poisson $\left(\mu_{\mathrm{i}}\right)$, where

$\log \mu_{\mathrm{i}}=\log E_{\mathrm{i}}+\beta^{\prime} \mathrm{x}_{\mathrm{i}}+\gamma_{0} \times$ year $_{\mathrm{i}}+\gamma_{1} \times$ alcohol density $_{\mathrm{i}}$ $+\gamma_{2} \times \%$ offsurrender $r_{\mathrm{i}}+\gamma_{3} \times$ indyear $_{\mathrm{i}} \times \%$ offsurrender $r_{\mathrm{i}}$ $+\phi_{\text {year }}, C_{i}$

where $\phi_{\text {year }}, \mathrm{C}_{i}$ is the spatial error term used to control the spatial relationship for the census tracts, in which "year" takes values from 1990 to 1999, and $C$ indexes the 290 census tracts. In the model, $\mathbf{x}$ is the vector of "nuisance" covariates ("\% Black", “\% Hispanic”, “\% Asian”, \% Male 15 to 30”, “\% Households in poverty" and "Damage per Square Mile") with corresponding coefficient $\beta$. We separately list the coefficients of variables of greater interest (year, alcohol density, \% offsale surrender, and the dual changepoint interaction term) as $\gamma$ is in the model.

To account for the potential spatial similarity across neighbourhoods, we employ a spatial smoothing analysis. We use the conditionally autoregressive (CAR; Besag, 1974) model as a prior distribution for the spatial random effects, so that 
the census tracts that are adjacent to each other are correlated. Given the other spatial error terms, the prior mean of the spatial error in tract $j$ equals the average error in the tracts that are adjacent to $\mathrm{j}$. Let “ $j \sim i$ ” denote adjacency of regions $i$ and $j$, and $n_{j}$ be the number of tracts adjacent to tract $j$. The CAR prior distribution of $\phi_{\text {year }}, C_{i}$ has the form

$$
\phi_{\text {year }_{i}, C_{i}=j} \mid \phi_{\text {year }_{i}, C_{i} \neq j} \sim N\left(\sum_{k-j} \frac{1}{n_{j}} \phi_{\text {year }_{i}, k}, \frac{1}{n_{j} \tau_{\text {year }_{i}}}\right)
$$

where $\tau_{\text {year }}$ is the precision parameter controlling the degree of spatial smoothing in year $\mathrm{i}$. The $\tau_{\text {year }_{i}}$ are smoothed temporally via a moderately informative gamma hyperprior distribution having mean 1 and variance 10. Non-informative prior distributions (i.e., normal distributions centered at 0 with precision 0.00001 ) are assumed for all other coefficients $\beta$ and $\gamma$.

Our Bayes approach obtains posterior distributions for all parameters of interest via Markov chain Monte Carlo (MCMC) algorithms implemented in WinBUGS (free software available at http://www.mrc-bsu.cam.ac.uk/bugs/welcome.shtml); Appendix 1 shows our WinBUGS code.

To compare models, we use the Deviance Information Criterion (DIC; Spiegelhalter et al., 2002). DIC not only measures the fit of models but also accounts for their complexity. The DIC generalises the Akaike Information Criterion (AIC) to hierarchical models. DIC is defined as

$$
D I C=\bar{D}+p_{D}
$$

where $\bar{D}$ is the posterior mean deviance, or minus twice the log-likelihood averaged over the MCMC samples, and $p_{D}$ is the posterior expectation of the deviance minus the deviance evaluated at the posterior means of the parameters. Spiegelhalter et al. (2002) show that $p_{D}$ captures the effective number of model parameters after accounting for the local shrinkage of the spatial random effects. Since we prefer models that fit well (small $\bar{D})$ yet are also of manageable size (small $p_{D}$ ), we generally choose the model having the smallest DIC score, where a difference of 3 to 5 units is normally thought of as the smallest worth mentioning.

\section{Results}

We completed 14 separate WinBUGS runs, one for each of the 14 definitions of "indyear" listed in Table 1. In each case, we used 500 iterations for MCMC burn-in, followed by a further 2000 iterations to determine the posterior estimates. Without loss of generality, we center all the covariates at 0 to reduce possible collinearities among variables. Table 2 presents the DIC and $p_{D}$ values for each of the 14 models. Note that since only the ranks of the DIC are important, we subtract 20000 from all the DIC for ease of comparison. From the table, we see that the values of $p_{D}$ remain relatively constant and fairly close to the theoretical upper bound of 2900 (the number of data points). This means that there is significant spatial variability left unexplained by all of our models. The DIC values suggest that Model 13 is the most sensible to use in evaluating the impact of alcohol license surrender on the assault rates following the civil unrest. According to this model, the effect of license surrender was lagged by one year, and then lasted for another 5 years.

Selecting Model 13 as the final model for further exploration, Table 3 presents the posterior distributions of the coefficients with the significant coefficients highlighted in grey. Since we modelled the covariates on the log scale, the "unit change" column converts back to the original scale, giving the multiplicative effect on the relative risk of assault per unit change in the corresponding covariate. We see a positive association between alcohol availability and assault, since the coefficient for alcohol outlet density is significantly positive. For example, if for a particular year census tract $\mathrm{A}$ has one more alcohol outlet per square mile than does tract B, and all other factors for the tracts are the same, then in that year we expect the relative risk of assault in tract A to be 1.062 times that of tract $\mathrm{B}$. The signif- 
icant negative coefficient for "year" indicates that the assault relative risk decreased by roughly $4.5 \%$ per year from 1990 to 1999 . Also, tracts that surrendered a greater percentage of their alcohol licenses in 1992 tended to have significantly higher assault risks, reflecting the fact that surrender tracts had higher assault rates on average right from the start of the study period. Census tracts with relatively more blacks, Hispanics or Asians, as well as poorer tracts, tend to have higher risks. Finally, the coefficient for the interaction term "indyear \% offsale surrender" is significant and negative, meaning that beginning one year after the civil unrest, on average, the census tracts that have relatively more license surrenders experienced more dramatic decreases in the assault rate, and this decrease was roughly $100-\exp (-0.256 * 0.01)=0.26 \%$ per year per percent increase in offsale surrender.

Table 4 shows the posterior means of the s, where larger values indicate greater residual spatial similarity. While they are all fairly similar, the residual spatial similarity among neighbours becomes slightly stronger over the years until 1997, perhaps the effect of the temporal transition period in our model.

Table 2. Model selection (DIC) and effective size $\left(p_{\mathrm{D}}\right)$ summaries.

\begin{tabular}{ccc|ccc}
\hline Model & DIC & $p_{\mathrm{D}}$ & Model & DIC & \multicolumn{1}{c}{$p_{\mathrm{D}}$} \\
\hline 1 & 1169.8 & 2353.05 & 8 & 1038.0 & 2367.51 \\
2 & 1136.6 & 2357.04 & 9 & 1152.0 & 2355.45 \\
3 & 1172.1 & 2354.12 & 10 & 1147.3 & 2358.64 \\
4 & 1169.5 & 2354.91 & 11 & 1084.5 & 2359.33 \\
5 & 1153.6 & 2356.33 & 12 & 1042.6 & 2364.41 \\
6 & 1107.0 & 2360.73 & 13 & 973.7 & 2367.52 \\
7 & 1035.3 & 2364.76 & 14 & 992.7 & 2369.79 \\
\hline
\end{tabular}

Table 3. Inferences for Model 13. The covariates that are significantly different from zero in the model are highlighted in grey. Note that the range for the variables with the prefix "\%" is actually from 0 to 1 rather than 0 to 100 .

\begin{tabular}{lrrrrrr}
\hline \multicolumn{1}{c}{ Coefficients } & Mean & Unit change & SD & Median & $2.5 \%$ & $97.5 \%$ \\
\hline Intercept & -0.210 & 0.8105 & 0.0081 & 0.2100 & -0.2254 & -0.1941 \\
\% Black & 1.709 & 5.5234 & 0.1325 & 1.7070 & 1.4230 & 1.9720 \\
\% Hispanic & 1.063 & 2.8950 & 0.0861 & 1.0590 & 0.8886 & 1.2240 \\
\% Asian & 0.824 & 2.2805 & 0.1381 & 0.5314 & 0.8259 & 1.0870 \\
\% Male 15 to 30 & -0.186 & 0.8296 & 0.1735 & 0.1879 & -0.5170 & 0.1531 \\
\% Household in poverty & 0.107 & 1.1130 & 0.0117 & 0.1070 & 0.0850 & 0.1298 \\
Damage per square mile & 0.000 & 1.0002 & 0.0003 & 0.0003 & -0.0004 & 0.0008 \\
Year & -0.045 & 0.9556 & 0.0018 & 0.0454 & -0.0502 & -0.0408 \\
Alcohol Density & 0.060 & 1.0620 & 0.0054 & 0.0602 & 0.0498 & 0.0705 \\
\% offsale surrender in 1992 & 0.399 & 1.4914 & 0.0671 & 0.4025 & 0.2600 & 0.5262 \\
Indyear x \%offsale surrender & -0.256 & 0.7741 & 0.0201 & -0.2924 & -0.2570 & -0.2130 \\
\hline
\end{tabular}

Table 4. Posterior means of $\tau$ by year; larger $\tau$ means higher similarity in the spatial residuals for that year.

\begin{tabular}{ccccccccccc}
\hline Year & 1990 & 1991 & 1992 & 1993 & 1994 & 1995 & 1996 & 1997 & 1998 & 1999 \\
\hline$\tau$ & 0.7 & 0.8 & 1 & 1.1 & 1.2 & 1.2 & 1.3 & 1.4 & 1.1 & 0.9 \\
\hline
\end{tabular}


Figure 2 maps the distribution of the fitted assault rates in 1999 in the "1992 Civil Unrest" area. The upper panels of Figure 2 separately map the original and the fitted assault rates, i.e. the number of assault crimes per 1000 census tract residents. The maps show reasonably good fit to the original data. They also reveal a concentration of assaults in the middle to east areas, which com- prise downtown LA and its immediate neighbourhood. The maps show that the north is relatively "safer" than the south. The lower panels map residuals, where the left panel is the fitted spatial error $\left(\phi_{1999_{i}, C_{i}}\right.$ in model $\left.(1)\right)$ and the right panel is the residuals after the model fitting, i.e., the raw assault rate minus the fitted assault rate at each census tract in 1999.

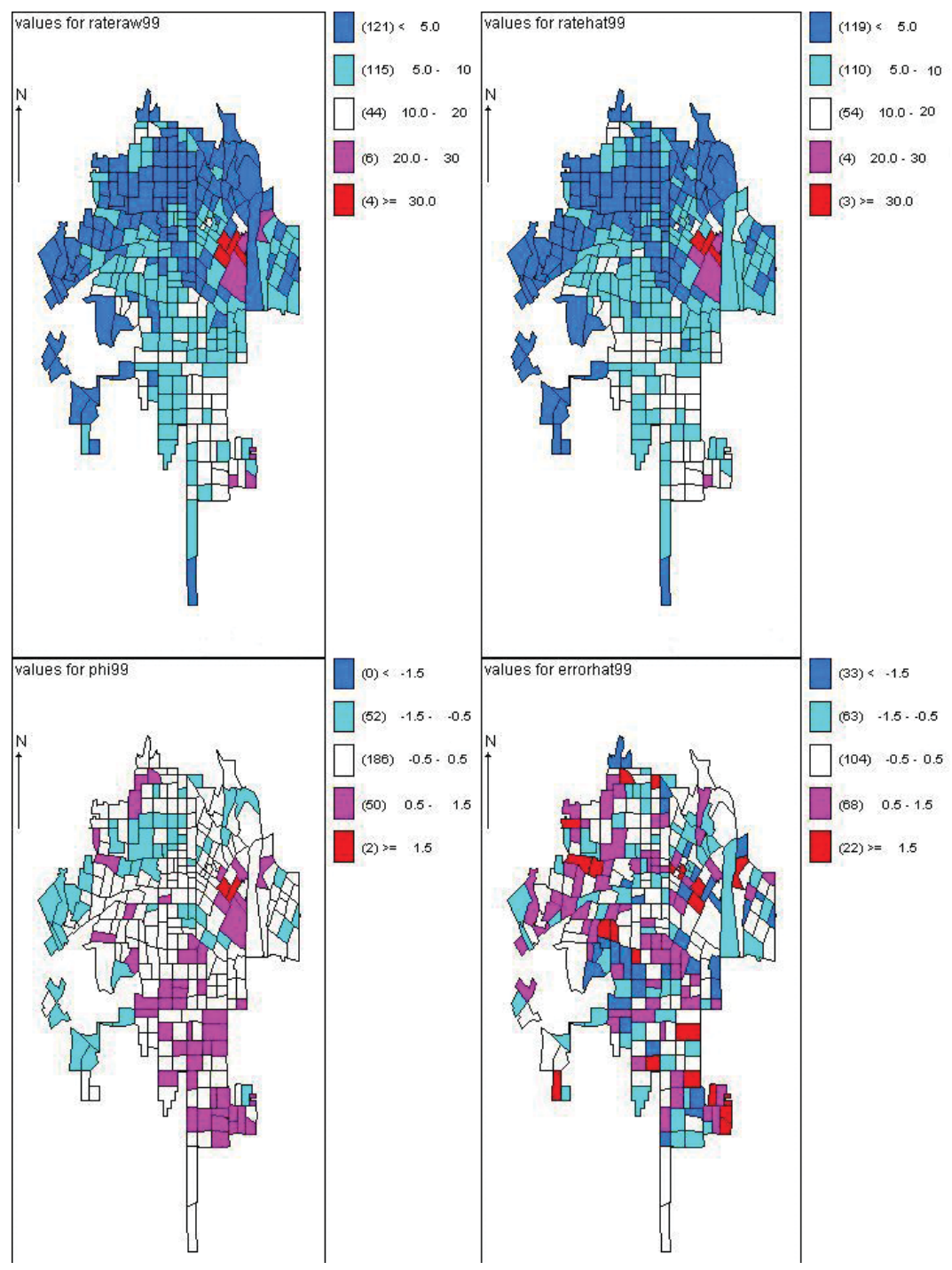

Fig. 2. Maps of assault crimes on original and fitted data. The left upper map is the observed assault rate in 1999, and the right upper panel maps the fitted assault rate. The left lower panel maps the fitted spatial error (note it is in the log scale) and the right lower panel shows the final residuals after taking out the spatial errors. 


\section{Discussion}

The literature on the contextual effects of the physical availability of alcohol in neighbourhood environments is at a stage in which researchers are advocating polices to change the alcohol environment to address alcohol related outcomes like assaultive violence. Given the difficulty in implementing such policies, natural experiments like the destruction of alcohol outlets that occurred during the "1992 Civil Unrest" are of particular importance for both researchers and policymakers (Cohen et al., 2003, 2006). However, the spatio-temporal structure of the data presents challenges for modeling such data. The changepoint model described represents one approach that essentially compares the trend in assault rates over time across census tracts which varied in terms of their loss of alcohol outlets in the civil unrest while simultaneously controlling for the possibility that levels of assault in neighboring tracts could affect trends in index tracts.

Taking into account other factors that might have an influence on the assault rate and utilizing a CAR model to smooth the spatial heterogeneity, we have demonstrated that the reduction in alcohol outlets following the "1992 Civil Unrest" in Los Angeles was positively associated with a decrease in assaultive violence at the census tract level. This association is demonstrated not only through the significant positive coefficient for the alcohol outlet density in our model, but also through the significant negative coefficient for the "indyear - \% offsale surrender" interaction term, which indicates that census tracts experienced an extra decrease in assaultive violence proportional to their level of alcohol license surrender. We did experiment with models that included a "year - \% offsale surrender" interaction, which would have implied different precivil unrest slopes for the two groups in Figure 1. However, the fitted interactions in these models were not significant.

Maps are provided to show the distribution of raw and fitted assault rates. Indeed, the residual maps in Figure 2 are potentially quite useful in extending our modeling, because the remaining spatial autocorrelation in the residuals after model fitting suggests the presence of unmeasured spatially varying covariates. One obvious candidate here would be the distance of each tract from the origin of the civil unrest (the intersection of Florence and Normandy). Additional possible factors are more social in nature, and are related to the fact that the southern region of the "1992 Civil Unrest" area tends to be the most disadvantaged in the city. While measures of SES and ethnicity are already included in the model, other factors associated with concentrated disadvantage (i.e., family structure, ethnic isolation, low social capital) were not but may contribute to the observed patterns.

Our analysis also used a linear model for the log-relative risk, and did not make any transformations of the potential explanatory variables. In practice, however, these assumptions may not be entirely appropriate. For example, exploratory analyses suggest that the assault rate initially decreases and then increases as the proportion of Asian residents in the neighbourhood increases. Without transforming the variables or adding a quadratic or other nonlinear term, this kind of relationship cannot be accommodated. We cannot transform the variables unless we are confident that the transformed variables would be linearly associated with the log-relative risk of response. A potential direction for future research here is to use MART (Multiple Additive Regression Trees; Friedman and Hall, 2000; Friedman, 2002) to build such a nonlinear relationship. A CAR model could still be incorporated to smooth residual spatial heterogeneity. We also hope to explore the relationship between alcohol availability and other forms of violent crime, such as homicide, rape, and armed robbery. A final area of interest is to study the association between alcohol availability and mortality rates in the study region.

\section{Appendix 1. Model written in WinBUGS}

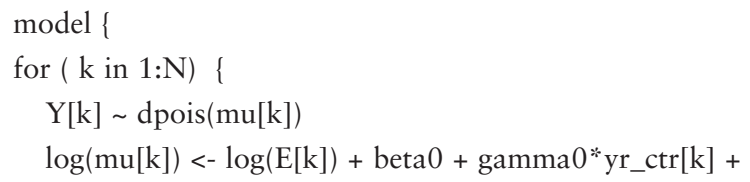




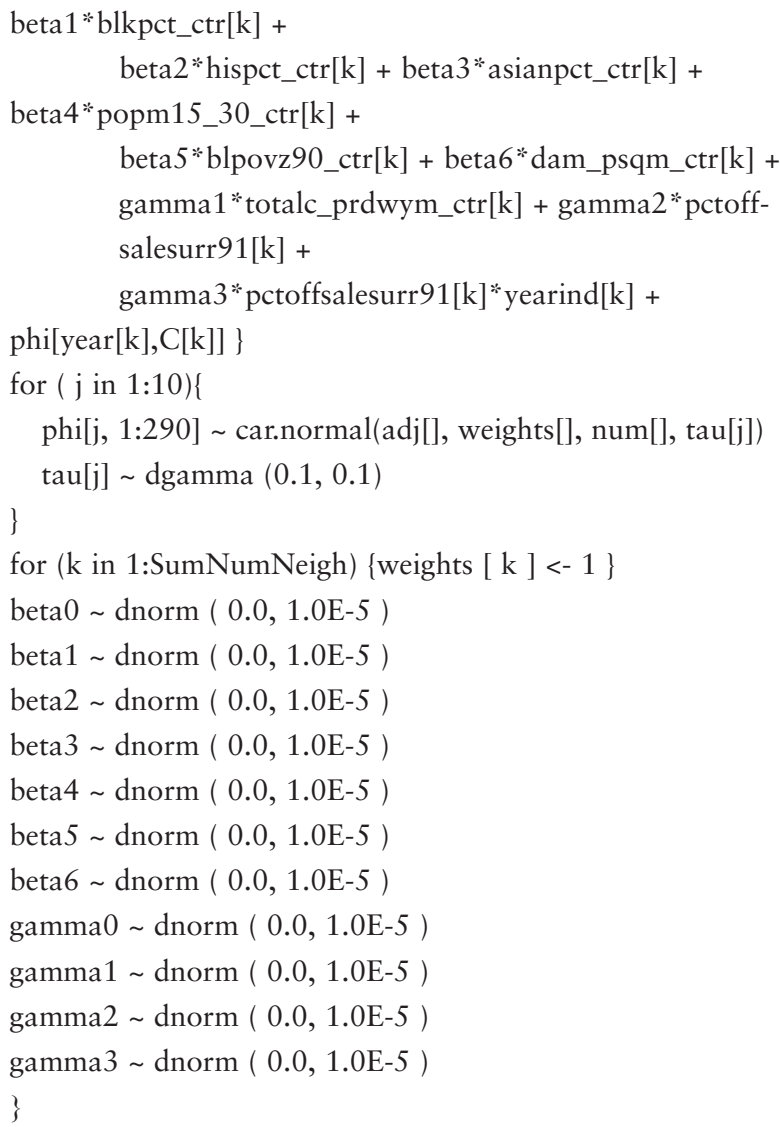

\section{References}

Berenstein L, 1994. Rebuilding rules prevail merchants must hire guards, shorten hours if they want to sell liquor. Los Angeles, USA, LA Times, B3.

Besag J, 1974. Spatial interaction and the statistical analysis of lattice systems (with discussion). J R Stat Soc Ser B 36, 192-236.

Besag J, York J, Mollie A, 1991. Bayesian image restoration, with two applications in spatial statistics. Ann Inst Stat Math 43, 1-59.

Britt HR, Carlin BP, Toomey T, Wagenaar A, 2005. Neighbourhood level spatial analysis of the relationship between alcohol outlet density and criminal violence. Env Ecol Stat 12, 411-426.

Carlin BP, Gelfand AE, Smith A, 1992. Hierarchical Bayesian analysis of changepoint problems. Appl Stat 41, 389-405.

Cohen D, Ghosh-Dastidar B, Scribner R, Miu A, Scott M,
Robinson P, Farley TA, Bluthenthal RN, Brown-Taylor D, 2006. Alcohol outlets, gonorrhea, and the Los Angeles civil unrest: a longitudinal analysis. Soc Sci Med 62, 3062-3071.

Cohen DA, Mason K, Bedimo A, Scribner R, Basolo V, Farley TA, 2003. Neighborhood physical conditions and health. Am J Public Health 93, 467-471.

Evans C, 1993. Public health impact of the 1992 Los Angeles civil unrest. Public Health Rep 108, 265-272.

Friedman JH, 2002. Stochastic gradient boosting. Comput Stat Data An 38, 367-378.

Friedman JH, Hall P, 2000. On bagging and nonlinear estimation. J Stat Plan Infer 137, 669-683.

Gorman DM, Speer PW, Gruenewald PJ, Labouvie EW, 2001. Spatial dynamics of alcohol availability, neighborhood structure and violent crime. J Stud Alcohol 62, 628-636.

Green P, 1995. Reversible jump Markov chain Monte Carlo computation and Bayesian model determination. Biometrika 85, 711-732.

Grills C, Bass K, Brown D, Akers A, 1996. Empowerment evaluation: building upon a tradition of activism in the African American community. In: Empowerment evaluation: knowledge and tools for self assessment and accountability. D Fetterman, S Daftarian, A Wandersman (eds). Thousand Oaks, CA, USA, Sage Publications, pp. 123-140. Gruenewald PJ, 2000. Small area estimates from a geostatisical perspective: current status and future directions. In: The epidemiology of alcohol problems in small geographic areas. Wilson and Dufour (eds), pp. 245-250.

Gruenewald PJ, Remer L, 2006. Changes in outlet densities affect violence rates. Alcohol Clin Exp Res 30, 1184-1193.

Hubler S, 2002. Extensive survey of riot area finds some surprises. Los Angeles, USA, LA Times, 1.

Kang C, 1994. Store owners to fight restrictions on reopening business. Los Angeles, USA, LA Times, B3.

Ong P, 1993. Losses in the Los Angeles Civil Unrest, April 29May 1, 1992: lists of the damaged properties and Korean merchants and the L.A. Riot Rebellion. UCLA, Los Angeles, USA. Scribner R, Cohen D, Kaplan S, Allen SH, 1999. Alcohol availability and homicide in New Orleans: conceptual considerations for small area analysis of the effect of alcohol outlet density. J Stud Alcohol 60, 310-306.

Scribner RA, MacKinnon DP, Dwyer JH, 1995. The risk of assaultive violence and alcohol availability in Los Angeles County. Am J Public Health 85, 335-340. 
Speer PW, Gorman DM, Labouvie EW, Ontkush MJ, 1998. Violent crime and alcohol availability: relationships in an urban community. J Public Health Policy 19, 303-318.

Spiegelhalter DJ, Best NG, Carlin BP, Linde A, 2002. Bayesian measures of model complexity and fit. J R Stat Soc Ser B 64, 583-639.

Watts RK, Rabow J, 1983. Alcohol availability and alcohol- related problems in 213 California cities. Alcohol Clin Exp Res 7, 47-58.

Zhu L, Gorman DM, Horel S, 2004. Alcohol outlet density and violence: a geospatial analysis. Alcohol Alcohol 39, 369-375. Zhu L, Gorman DM, Horel S, 2006. Hierarchical Bayesian spatial models for alcohol availability, drug "hot spots" and violent crime. Int J Health Geogr 5, 54. 